\title{
Gevrey Regularity and Time Decay of Fractional Porous Medium Equation in Critical Besov Spaces
}

\author{
Weiliang Xiao, Yu Zhang* \\ School of Applied Mathematics, Nanjing University of Finance and Economics, Nanjing, China \\ Email: xwltc123@163.com, *yuzhang952020@163.com
}

How to cite this paper: Xiao, W.L. and Zhang, Y. (2022) Gevrey Regularity and Time Decay of Fractional Porous Medium Equation in Critical Besov Spaces. Journal of Applied Mathematics and Physics, 10, 91-111.

https://doi.org/10.4236/jamp.2022.101008

Received: December 5, 2021

Accepted: January 16, 2022

Published: January 19, 2022

Copyright $\odot 2022$ by author(s) and Scientific Research Publishing Inc. This work is licensed under the Creative Commons Attribution International License (CC BY 4.0).

http://creativecommons.org/licenses/by/4.0/

\section{(c) (i) Open Access}

\begin{abstract}
In this paper, we show the existence and regularity of mild solutions depending on the small initial data in Besov spaces to the fractional porous medium equation. When $1<\alpha \leq 2$, we prove global well-posedness for initial data $u_{0} \in \dot{B}_{p, r}^{-\alpha+\frac{n}{p}+2-2 m}\left(\mathbb{R}^{n}\right)$ with $1 \leq p<\infty, 1 \leq q \leq \infty$, and analyticity of solutions with $1<p<\infty, 1 \leq q \leq \infty$. In particular, we also proved that when $\alpha=1$, both $u$ and $\mathrm{e}^{t^{\frac{1}{2 n}} \Lambda_{1}} u$ belong to $\mathcal{X}_{T^{*}}$. We solve this equation through the contraction mapping method based on Littlewood-Paley theory and Fourier multiplier. Furthermore, we can get time decay estimates of global solutions in Besov spaces, which is $t^{-\frac{1}{\alpha}}$ as $t \rightarrow \infty$.
\end{abstract}

\section{Keywords}

Well-Posedness, Gevrey Regularity, Time Decay, Besov Spaces

\section{Introduction}

In this paper, we consider existence and regularity of mild solutions for the initial value problem of the following fractional porous medium equation (FPME) in $\mathbb{R}^{n}$ for $n \geq 2$ :

$$
\begin{cases}\partial_{t} u+\gamma \Lambda^{\alpha} u+\nabla \cdot(u \nabla P u)=0 & \text { in } \mathbb{R}^{n} \times(0, \infty), \\ P u=\kappa(-\Delta)^{-m} u & \text { in } \mathbb{R}^{n} \times(0, \infty), \\ u(x, 0)=u_{0}(x) & \text { in } \mathbb{R}^{n},\end{cases}
$$

where $u$ and $P u$ denote the density and gas pressure respectively, while 
$u_{0}=u_{0}(x)$ denotes the given initial data. And $\kappa= \pm 1$. The positive operator $\Lambda^{\alpha}=(-\Delta)^{\frac{\alpha}{2}}$ can be defined by

$$
\Lambda^{\alpha} f(x):=c(\alpha, n) P . V \cdot \int_{\mathbb{R}^{n}} \frac{f(x)-f(y)}{|x-y|^{n+\alpha}} \mathrm{d} y,
$$

where $c(\alpha, n)$ is a normalization constant. By the Fourier transform, we can also get a very simple alternative representation, as $\Lambda^{\alpha} f=\mathcal{F}^{-1}\left[|\xi|^{\alpha} \mathcal{F} f(\xi)\right]$, where $\mathcal{F}$ is the Fourier transform and $\mathcal{F}^{-1}$ is the inverse Fourier transform, respectively. To simplify the notation, we consider $\gamma=\kappa=1$. For FPME research, there have been many results. For example, in 2019, Feng and Liu [1] used generalized Riccati transformation technique and the differential inequality method to obtain the oscillation criteria of a class of nonlinear fractional differential equations.

If $\gamma>0, \kappa=1, \alpha=2, m=1$, the system (1) reduces to the classical Keller-Segel model

$$
\begin{cases}\partial_{t} u+\gamma(-\Delta) u+\nabla \cdot(u \nabla P u)=0 & \text { in } \mathbb{R}^{n} \times(0, \infty), \\ P u=\kappa(-\Delta) u & \text { in } \mathbb{R}^{n} \times(0, \infty), \\ u(x, 0)=u_{0}(x) & \text { in } \mathbb{R}^{n} .\end{cases}
$$

The Keller-Segel system has been established as the model of chemotaxis by Keller and Segel [2]. Biler and Karch [3] demonstrated local and global solutions with small initial data of the equation in critical Lebesgue space $L^{\bar{\alpha}}$ for $1<\alpha<2$. Biler and $\mathrm{Wu}[4]$ studied global well-posedness of the equation with small initial data in the critical Besov spaces $\dot{B}_{2, q}^{1-\alpha}\left(\mathbb{R}^{2}\right)$ for $1<\alpha<2$. Zhao, Cui and Liu [5] proved small data global existence and large data local existence of solutions in critical Besov space $\dot{B}_{p, q}^{-2+\frac{1}{p}}$ for $1<p<2 n$ and $1 \leq q \leq \infty$.

The purpose of this paper is to prove the well-posedness and Gevrey analyticity of Equation (1) in the Besov spaces. When $\gamma=0, \kappa=-1, m=0$, the equation degenerates to the classic form of the Porous Media Equation. We refer it by PME, and the classical properties of this equation can be found in literature [6]. It can describe the movement of an ideal gas flowing through a porous medium or be regarded as a kind of non-local quadratic evolution problem. This equation is widely used in describing Brownian diffusion, gas particle interaction, and biotaxis. When $\gamma=0, \kappa=-1,0<m<1$, the equation was first proposed by Caffarelli and Vázquez [7]. It has been proved by them that, when the initial value $u_{0}$ is a bounded function and exponentially decays at infinity, the equation has a weak solution. In [8], they studied the $C^{\beta}$ regularity of the weak solution of the equation when $\gamma=0, \kappa=-1$. For the detailed information about the solution of this equation, please refer to literature [9].

Secondly, we present some results of Gevrey analyticity in recent years. In 1989, Foias and Temam discovered this method and employed it for the first time to study the analyticity of the Navier-Stokes equations with space periodicity boundary condition, see [10] [11]. In the following periods, a few more authors 
made full use of this method, and extended it to various functional spaces and equations. For instance, in 2001, Zhan [12] obtained the Gevrey regularity of the solution of the superconducting phase-locked equation. Ferrari and Titi in [13] studied the regularity of solutions to a large class of analytic nonlinear parabolic equations on the two-dimensional sphere. Chueshov and Polat in [14] studied the Gevrey regularity of the global attractor of the dynamical system generated by the generalized Benjamin-Bona-Mahony equation with periodic boundary conditions. Recently, the well-posedness and spatial regularity of the classic K-S equation have been completed by Zhao [15].

In this paper, we will consider well-posedness and Gevrey analyticity of the fractional porous medium Equation (1) with initial data in critical Besov spaces $\dot{B}_{p, r}^{-\alpha+\frac{n}{p}+2-2 m}$ for $1 \leq \alpha \leq 2$ and $1 \leq p, r \leq \infty$. To address the equations, we consider the following integral equations:

$$
u(t)=\mathrm{e}^{-t \Lambda^{\alpha}} u_{0}-\int_{0}^{t} \mathrm{e}^{-(t-\tau) \Lambda^{\alpha}} \nabla \cdot\left(u \nabla(-\Delta)^{-m} u\right) \mathrm{d} \tau .
$$

where $\mathrm{e}^{-t \Lambda^{\alpha}}:=\mathcal{F}^{-1}\left(\mathrm{e}^{-t|\xi|^{\alpha}} \mathcal{F}\right)$. We use the following the contraction mapping to get the solution of (3):

$$
\mathbb{G}(u):=\mathrm{e}^{-t \Lambda^{\alpha}} u_{0}-\int_{0}^{t} \mathrm{e}^{-(t-\tau) \Lambda^{\alpha}} \nabla \cdot\left(u \nabla(-\Delta)^{-m} u\right) \mathrm{d} \tau .
$$

Then we utilize the Gevrey class regularity to certify analyticity of solutions. As an application, we get time decay rates in Besov spaces for global solutions.

Let us denote by $\Lambda_{1}$ the Fourier multiplier whose symbol is given by $|\xi|_{1}=\left|\xi_{1}\right|+\cdots+\left|\xi_{n}\right|$.

The overall structure of the article is shown below. In Section 2, we review the Littlewood-Paley dyadic decomposition theory and the definition of Besov spaces. In Section 3 and Section 4, we prove Theorem 3.3 and Theorem 3.5 respectively by the standard fixed point argument.

\section{Notations and Preliminaries}

First of all, let's introduce some notations mentioned in the paper. We will consider the solution of system (1) in $\mathbb{R}^{n}$. For two constants $A$ and $B$, if there is a finite constant $C$ whose value of each line may vary such that $A \leq C B$, we denote it as $A \lesssim B$. For a quasi-Banach space $X$ and for any $0<T \leq \infty$, we use standard notation $L^{p}(0, T ; X)$ to denote the quasi-Banach space of Bochner measurable functions $f$ from $(0, T)$ to $X$ endowed with the norm

$$
\|f\|_{L_{T}^{p} X}:= \begin{cases}\left(\int_{0}^{T}\|f(\cdot, t)\|_{X}^{p} \mathrm{~d} t\right)^{\frac{1}{p}} & \text { if } 1 \leq p<\infty, \\ \sup _{0 \leq t \leq T}\|f(\cdot, t)\|_{X} & \text { if } p=\infty .\end{cases}
$$

Especially, if $T=\infty$, we still use $\|f\|_{L_{T}^{p} X}$ rather than $\|f\|_{L_{\infty}^{p} X}$.

Let us introduce some basic knowledge on Littlewood-Paley theory and Besov spaces.

Let $\varphi \in C_{c}^{\infty}\left(\mathbb{R}^{n}\right)$ be a radial positive function such that 


$$
\operatorname{supp} \varphi \subset\left\{\xi \in \mathbb{R}^{n}: \frac{3}{4} \leq|\xi| \leq \frac{8}{3}\right\}, \quad \sum_{j \in Z} \varphi\left(2^{-j} \xi\right)=1 \text { for any } \xi \neq 0 .
$$

Define the frequency localization operators as follows:

$$
\Delta_{j} u=\varphi_{j}(D) u=\mathcal{F}^{-1} \varphi_{j}(\xi) \mathcal{F} u ; S_{j} u=\psi_{j}(D) u=\mathcal{F}^{-1} \psi_{j}(\xi) \mathcal{F} u
$$

here $\varphi_{j}(\xi)=\varphi\left(2^{-j} \xi\right)$ and $\psi_{j}=\sum_{k \leq j-1} \varphi_{j}$.

By Bony's decomposition, we can split the product $u v$ into three parts:

$$
u v=T_{u} v+T_{v} u+R(u, v)
$$

with

$$
T_{u} v=\sum_{j} S_{j-1} u \Delta_{j} v, R(u, v)=\sum_{j} \Delta_{j} u \tilde{\Delta}_{j} v, \tilde{\Delta}_{j} v=\Delta_{j-1} v+\Delta_{j} v+\Delta_{j+1} v .
$$

Let us now define the Besov spaces as follows.

Definition 2.1 For $s<\frac{n}{p}$ (or $s=\frac{n}{p}$ if $\left.r=1\right), s \in \mathbb{R}, p, r \in[1, \infty]$ and $f \in \mathcal{S}^{\prime}\left(\mathbb{R}^{n}\right)$, we define the homogeneous Besov space $\dot{B}_{p, r}^{s}$ as

$$
\dot{B}_{p, r}^{s}=\left\{f \in \mathcal{S}^{\prime}\left(\mathbb{R}^{n}\right) / \mathcal{P}\left(\mathbb{R}^{n}\right):\|f\|_{\dot{B}_{p, r}^{s}}=\left(\sum_{j \in \mathbb{Z}} 2^{j s r}\left\|\Delta_{j} f\right\|_{L^{p}}^{r}\right)^{1 / r}<\infty\right\} .
$$

Here the norm changes normally when $p=\infty$ or $r=\infty$, and $\mathcal{P}$ is the set of all polynomials. If $k \in \mathbb{N}$ and $\frac{n}{p}+k \leq s \leq \frac{n}{p}+k+1 \quad$ (or $s=\frac{n}{p}+k+1$ if $r=1)$, then $\dot{B}_{p, r}^{s}$ is defined as the subset of distributions $f \in \mathcal{S}^{\prime}\left(\mathbb{R}^{n}\right)$ so that $\partial^{\beta} f \in \dot{B}_{p, r}^{s-k}$ when $|\beta|=k$.

Definition 2.2 For $0<T \leq \infty, s \leq \frac{n}{p}(s \in \mathbb{R}), 1 \leq p, r, \rho \leq \infty$, we set (with the usual convention if $r=\infty$ )

$$
\|f\|_{\tilde{L}_{T}^{\rho}\left(\dot{B}_{p, r}^{s}\right)}:=\left(\sum_{j \in Z} 2^{j s r}\left\|\Delta_{j} f\right\|_{L^{\rho}\left(0, T ; L^{p}\right)}^{r}\right)^{\frac{1}{r}} .
$$

We then define the space $\tilde{L}^{\rho}\left(0, T ; \dot{B}_{p, r}^{s}\left(\mathbb{R}^{n}\right)\right)$ as the set of temperate distributions $f$ over $(0, T) \times \mathbb{R}^{n}$ such that $\lim _{j \rightarrow-\infty} S_{j} f=0$ in $S^{\prime}\left((0, T) \times \mathbb{R}^{n}\right)$ and $\|f\|_{\tilde{L}_{T}^{\rho}\left(\dot{B}_{p, r}^{s}\right)}<\infty$.

Lemma 2.3 [16] Let $\mathcal{B}$ be a ball, and $\mathcal{C}$ a ring in $\mathbb{R}^{n}$. There exists a constant $C$ such that for positive real number $\lambda$, any nonnegative integer $k$ and any couple of real numbers $(p, r)$ with $1 \leq p \leq r \leq \infty$, we know

$$
\begin{aligned}
& \operatorname{supp} \mathcal{F}(f) \subset \lambda \mathcal{B} \Rightarrow \sup _{|\alpha|=k}\left\|\partial^{\alpha} f\right\|_{L^{r}} \leq C^{k+1} \lambda^{k+n\left(\frac{1}{p}-\frac{1}{r}\right)}\|f\|_{L^{p}}, \\
& \operatorname{supp} \mathcal{F}(f) \subset \lambda \mathcal{C} \Rightarrow C^{-k-1} \lambda^{k}\|f\|_{L^{p}} \leq \sup _{|\alpha|=k}\left\|\partial^{\alpha} f\right\|_{L^{r}} \leq C^{k+1} \lambda^{k}\|f\|_{L^{p}} .
\end{aligned}
$$

Lemma 2.4 [16] Let $f$ be a smooth function on $\mathbb{R}^{n} \backslash\{0\}$ which is homoge- 
neous of degree $m$. Then for any $s \in \mathbb{R}, 1 \leq p, q \leq \infty$, and $s-m<\frac{n}{p}$, or $s-m=\frac{n}{p}$ and $q=1$, the operator $f(D)$ is continuous from $\dot{B}_{p, q}^{s}\left(\mathbb{R}^{n}\right)$ to $\dot{B}_{p, q}^{s-m}\left(\mathbb{R}^{n}\right)$.

Lemma 2.5 [17] Let $\mathcal{C}$ be a ring in $\mathbb{R}^{n}$. There exist two positive constants $\kappa$ and $\mathcal{K}$ such that for any $p \in[1, \infty]$ and any couple $(t, \lambda)$ of positive real numbers, we have

$$
\operatorname{supp} \mathcal{F}(f) \subset \lambda \mathcal{C} \Rightarrow\left\|\mathrm{e}^{t \Lambda^{\alpha}} f\right\|_{L^{p}} \leq \mathcal{K} \mathrm{e}^{-\kappa \lambda^{\alpha} t}\|f\|_{L^{p}} .
$$

\section{The Case $1<\alpha \leq 2$ : Well-Posedness, Regularity and Time Decay}

In this chapter we will demonstrate well-posedness and Gevrey analyticity of Equation (1) in critical Besov spaces $\dot{B}_{p, r}^{-\alpha+\frac{n}{p}+2-2 m}$ for $1<\alpha \leq 2$. Let $n \geq 2$, $1<\alpha \leq 2$. Suppose $u_{0} \in \dot{B}_{p, r}^{-\alpha+\frac{n}{p}+2-2 m}\left(\mathbb{R}^{n}\right)$. When $1 \leq p, r \leq \infty$, let us prove the above properties.

\subsection{The Case $1 \leq p<\infty$ : Well-Posedness}

First of all, we give a priori estimate for the following fractional dissipative equation:

$$
\begin{cases}\partial_{t} u+\Lambda^{\alpha} u=f, & x \in \mathbb{R}^{n}, t>0, \\ u(x, 0)=u_{0}(x), & x \in \mathbb{R}^{n} .\end{cases}
$$

Proposition 3.1 ([4]) Let $s \in \mathbb{R}, 1 \leq p, r, \rho_{1} \leq \infty$ and $0<T \leq \infty$. There exists a constant $C>0$ depending only on $\alpha$ and $n$ such that for any $\rho_{1} \leq \rho \leq \infty$, we have

$$
\left.\|u\|_{\tilde{L}_{T}^{\rho}\left(\dot{B}_{p, r}^{s+\frac{\alpha}{\rho}}\right)} \leq C\left(\left\|u_{0}\right\|_{\dot{B}_{p, r}^{s}}+\|f\|_{\tilde{L}_{T}^{\rho_{1}}\left(\dot{B}_{p, r}^{s+\frac{\alpha}{\rho_{1}}}-\alpha\right.}\right)\right) .
$$

Next we present the following vital bilinear estimates.

Lemma 3.2 Let $s>0, \varepsilon>0,2 m-1+\varepsilon>0,1 \leq p<+\infty$, $1 \leq r, \rho, \rho_{1}, \rho_{2} \leq+\infty$ with $\frac{1}{\rho}=\frac{1}{\rho_{1}}+\frac{1}{\rho_{2}}$. There holds

$$
\left\|u \nabla(-\Delta)^{-m} v\right\|_{\tilde{L}_{T}^{\rho}\left(\dot{B}_{p, r}^{s}\right)} \lesssim\|u\|_{\dot{L}_{T}^{\rho_{1}}\left(\dot{B}_{p, r}^{s+\varepsilon}\right)}\|v\|_{\dot{L}_{T}^{\rho_{2}}\left(\dot{B}_{p, r}^{1-2 m+\frac{n}{p} \varepsilon}\right)} .
$$

Proof. According to Bony's paraproduct decomposition, we find that

$$
\begin{aligned}
u \nabla(-\Delta)^{-m} v= & \sum_{j^{\prime} \in Z} \Delta_{j^{\prime}} u \nabla(-\Delta)^{-m} S_{j^{\prime}-1}+\sum_{j^{\prime} \in Z} S_{j^{\prime}-1} u \nabla(-\Delta)^{-m} \Delta_{j^{\prime}} v \\
& +\sum_{j^{\prime} \in Z} \sum_{\mid j^{\prime}-j^{\prime \prime} \leq \leq 1} \Delta_{j^{\prime}} u \nabla(-\Delta)^{-m} \Delta_{j^{\prime \prime}} v \\
:= & I_{1}+I_{2}+I_{3} .
\end{aligned}
$$


Firstly, we estimate $I_{1}$. Applying Hölder's inequality and Lemmas 2.3, 2.4, there holds

$$
\begin{aligned}
& \left\|\Delta_{j} \sum_{j^{\prime} \in Z} \Delta_{j^{\prime}} u \nabla(-\Delta)^{-m} S_{j^{\prime}-1} v\right\|_{L_{T}^{\rho}\left(L^{p}\right)} \\
& \lesssim \sum_{\left|j-j^{\prime}\right| \leq 4}\left\|\Delta_{j^{\prime}} u\right\|_{L_{T}^{\rho_{1}}\left(L^{p}\right)}\left\|\nabla(-\Delta)^{-m} S_{j^{\prime}-1} v\right\|_{L_{T}^{\rho_{2}}\left(L^{\infty}\right)} \\
& \lesssim \sum_{\left|j-j^{\prime}\right| \leq 4}\left\|\Delta_{j^{\prime}} u\right\|_{L_{T}^{\rho_{1}}\left(L^{p}\right)} \sum_{k \leq j^{\prime}-2} 2^{k\left(1-2 m+\frac{n}{p}\right)}\left\|\Delta_{k} v\right\|_{L_{T}^{\rho_{2}}\left(L^{p}\right)} \\
& \lesssim \sum_{\left|j-j^{\prime}\right| \leq 4}\left\|\Delta_{j^{\prime}} u\right\|_{L_{T}^{\rho_{1}}\left(L^{p}\right)} \sum_{k \leq j^{\prime}-2} 2^{\varepsilon k} 2^{\left(1-2 m+\frac{n}{p}-\varepsilon\right) k}\left\|\Delta_{k} v\right\|_{L_{T}^{\rho_{2}}\left(L^{p}\right)} \\
& \lesssim \sum_{\left|j-j^{\prime}\right| \leq 4} 2^{-j^{\prime}} 2^{(s+\varepsilon) j^{\prime}}\left\|\Delta_{j^{\prime}} u\right\|_{L_{T}^{\rho_{1}}\left(L^{p}\right)}\|v\|_{\tilde{L}_{T}^{\rho_{2}}\left(\dot{B}_{p, r}^{1-2 m+\frac{n}{p}-\varepsilon}\right) .}
\end{aligned}
$$

Multiplying (10) by $2^{s j}$ and taking $l^{r}$-norm, we get

$$
\left\|I_{1}\right\|_{\tilde{L}_{T}^{\rho}\left(\dot{B}_{p, r}^{s}\right)} \lesssim\|u\|_{\tilde{L}_{T}^{\rho_{1}}\left(\dot{B}_{p, r}^{s+\varepsilon}\right)}\|v\|_{\tilde{L}_{T}^{\rho_{2}}\left(\dot{B}_{p, r}^{1-2 m+\frac{n}{p}-\varepsilon}\right)}
$$

Similarly, for $I_{2}$, utilizing Hölder's inequality and Lemmas 2.3, 2.4, when $2 m-1+\varepsilon>0$, we find that

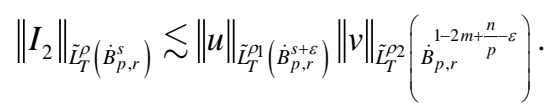

Now we deal with the term $I_{3}$. For one thing, we premeditate the case of $2 \leq p<\infty$, firstly using Bernstein's inequality, then by Hölder's inequality with $\frac{2}{p}=\frac{1}{p}+\frac{1}{p}$,

$$
\begin{aligned}
& \left\|\Delta_{j} \sum_{j^{\prime} \in Z} \sum_{j^{\prime}-j^{\prime \prime} \mid \leq 1} \Delta_{j^{\prime}} u \nabla(-\Delta)^{-m} \Delta_{j^{\prime \prime}} v\right\|_{L_{T}^{\rho}\left(L^{p}\right)} \\
& \lesssim 2^{\frac{n}{p} j} \sum_{j^{\prime} \geq j-N_{0}} \sum_{\left|j^{\prime}-j^{\prime \prime}\right| \leq 1} 2^{\left(-s-\frac{n}{p}\right) j^{j^{\prime}}} 2^{(s+\varepsilon) j^{\prime}}\left\|\Delta_{j^{\prime}} u\right\|_{L_{T}^{\rho_{1}}\left(L^{p}\right)} 2^{\left(1-2 m+\frac{n}{p}-\varepsilon\right) j^{\prime \prime}}\left\|\Delta_{j^{\prime \prime}} v\right\|_{L_{T}^{\rho_{2}}\left(L^{p}\right)} \\
& \lesssim 2^{-s j} \sum_{j^{\prime} \geq j-N_{0}} 2^{-\left(s+\frac{n}{p}\right)\left(j^{\prime}-j\right)} 2^{(s+\varepsilon) j^{\prime}}\left\|\Delta_{j^{\prime}} u\right\|_{L_{T}^{\rho_{1}}\left(L^{p}\right)}\|v\|_{\dot{L}_{T}^{p_{2}}\left(\dot{B}_{p, r}^{1-2 m+\frac{n}{p}-\varepsilon}\right)}
\end{aligned}
$$

For another case: $1 \leq p<2,2<p^{\prime} \leq \infty, \frac{1}{p}+\frac{1}{p^{\prime}}=1$, firstly using Bernstein's inequality, then by Hölder's inequality, and exploiting Bernstein's inequality again,

$$
\begin{aligned}
& \left\|\Delta_{j} \sum_{j^{\prime} \in Z} \sum_{\left|j^{\prime}-j^{\prime \prime}\right| \leq 1} \Delta_{j^{\prime}} u \nabla(-\Delta)^{-m} \Delta_{j^{n}} v\right\|_{L_{T}^{\rho}\left(L^{p}\right)} \\
& \lesssim 2^{j\left(n-\frac{n}{p}\right)} \sum_{j^{\prime} \geq j-N_{0}} \sum_{\left|j^{\prime}-j^{\prime \prime}\right| \leq 1} 2^{-\left(n-\frac{n}{p}+s\right)} 2^{(s+\varepsilon) j^{\prime}}\left\|\Delta_{j^{\prime}} u\right\|_{L_{T}^{\rho_{1}}\left(L^{p}\right)} 2^{\left(1-2 m+\frac{n}{p}-\varepsilon\right)}\left\|\Delta_{j^{\prime \prime}} v\right\|_{L_{T}^{\rho_{2}}\left(L^{p}\right)} \\
& \lesssim 2^{-s j} \sum_{j^{\prime} \geq j-N_{0}} 2^{-\left(n-\frac{n}{p}+s\right)\left(j^{\prime}-j\right)} 2^{(s+\varepsilon) j^{\prime}}\left\|\Delta_{j^{\prime}} u\right\|_{L_{T}^{\rho_{1}}\left(L^{p}\right)}\|v\|_{L_{L_{T}^{\rho_{2}}}\left(\dot{B}_{p, r}^{1-2 m+\frac{n}{p}}\right)}
\end{aligned}
$$


Under the assumption of Lemma 3.2, we have

$$
s+\frac{n}{p}>0, n-\frac{n}{p}+s>0 .
$$

Therefore we deduce from the estimate (13)-(14) that for all $1 \leq p<\infty$,

$$
\left\|I_{3}\right\|_{\tilde{L}_{T}^{\rho}\left(\dot{B}_{p, r}^{s}\right)} \lesssim\|U\|_{\tilde{L}_{T}^{\rho_{1}}\left(\dot{B}_{p, r}^{s+\varepsilon}\right)}\|V\|_{\tilde{L}_{T}^{\rho_{2}}}\left(\dot{B}_{p, r}^{1-2 m+\frac{n}{p} \varepsilon}\right) .
$$

Now we are in a position to prove well-posedness of the system (1) in the case that $1<\alpha \leq 2$ and $1 \leq p<\infty$.

Theorem 3.3 Let $1 \leq p<\infty, \quad \frac{2 \alpha-2}{\alpha}=\frac{1}{\rho_{1}}+\frac{1}{\rho_{2}}, \quad s=1-2 m+\frac{n}{p} \quad$ and $\varepsilon=1-\alpha+\frac{\alpha}{\rho_{1}}$. Then we have a $T^{*}=T^{*}\left(u_{0}\right)>0$ such that the system (1) has a unique solution $u \in \mathcal{X}_{T^{*}}$, where

$$
\mathcal{X}_{T^{*}}:=\tilde{L}^{\infty}\left(0, T^{*} ; \dot{B}_{p, r}^{-\alpha+\frac{n}{p}+2-2 m}\left(\mathbb{R}^{n}\right)\right) \cap \tilde{L}^{\rho_{1}}\left(0, T^{*} ; \dot{B}_{p, r}^{s_{1}}\left(\mathbb{R}^{n}\right)\right) \cap \tilde{L}^{\rho_{2}}\left(0, T^{*} ; \dot{B}_{p, r}^{s_{2}}\left(\mathbb{R}^{n}\right)\right)
$$

with

$$
s_{1}=\frac{n}{p}-\alpha+2-2 m+\frac{\alpha}{\rho_{1}}, s_{2}=\frac{n}{p}-\alpha+2-2 m+\frac{\alpha}{\rho_{2}}, \frac{1}{\rho_{1}}+\frac{1}{\rho_{2}}=\frac{1}{\rho} .
$$

If $T^{*}<\infty$, we have

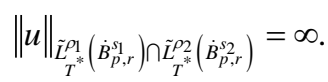

Furthermore, if the initial value $u_{0}$ is small enough in $\dot{B}_{p, r}^{-\alpha+\frac{n}{p}+2-2 m}\left(\mathbb{R}^{n}\right)$, then $T^{*}=\infty$.

Proof. Define the map

$$
\mathbb{G}: u(t) \rightarrow \mathrm{e}^{-t \Lambda^{\alpha}} u_{0}-\int_{0}^{t} \mathrm{e}^{-(t-\tau) \Lambda^{\alpha}} \nabla \cdot\left(u \nabla(-\Delta)^{-m} u\right) \mathrm{d} \tau .
$$

in the metric space $(I=[0, T])$ :

$$
\mathscr{V}_{T}:=\left\{u:\|u\|_{\tilde{L}_{T}^{\rho_{1}}\left(\dot{B}_{p, r}^{s_{1}}\right) \cap \tilde{L}_{T}^{\rho_{2}}\left(\dot{B}_{p, r}^{s_{2}}\right)} \leq \eta, d(u, v):=\|u-v\|_{\tilde{L}_{T}^{\rho_{1}}\left(\dot{B}_{p, r}^{s_{1}}\right) \cap \tilde{L}_{T}^{\rho_{2}}\left(\dot{B}_{p, r}^{s_{2}}\right)}\right\}
$$

with

$$
s_{1}=\frac{n}{p}-\alpha+2-2 m+\frac{\alpha}{\rho_{1}}, s_{2}=\frac{n}{p}-\alpha+2-2 m+\frac{\alpha}{\rho_{2}} .
$$

Exploiting Proposition 3.1 and Lemma 3.2 by choosing $\rho=\frac{\alpha}{2 \alpha-2}$, for any $u, v \in \mathscr{V}_{T}$, we get

$$
\begin{aligned}
& \|\mathbb{G}(u)\|_{\tilde{L}_{T}^{\rho_{1}}\left(\dot{B}_{p, r}^{s_{1}}\right) \cap \tilde{L}_{T}^{\rho_{2}}\left(\dot{B}_{p, r}^{s_{2}}\right)} \\
& \lesssim\left\|\mathrm{e}^{-t \Lambda^{\alpha}} u_{0}\right\|_{\tilde{L}_{T}^{\rho_{1}}\left(\dot{B}_{p, r}^{s_{1}}\right) \cap \tilde{L}_{T}^{\rho_{2}}\left(\dot{B}_{p, r}^{s_{2}}\right)}+\left\|u \nabla(-\Delta)^{-m} u\right\|_{\tilde{L}_{T}^{2 \alpha-2}\left(\dot{B}_{p, r}^{1-2 m+\frac{n}{p}}\right)} \\
& \lesssim\left\|\mathrm{e}^{-t \Lambda^{\alpha}} u_{0}\right\|_{\tilde{L}_{T}^{\rho_{1}}\left(\dot{B}_{p, r}^{s_{1}}\right) \cap \tilde{L}_{T}^{\rho_{2}}\left(\dot{B}_{p, r}^{s_{2}}\right)}+\|u\|_{\tilde{L}_{T}^{\rho_{1}}\left(\dot{B}_{p, r}^{s_{1}}\right) \cap \tilde{L}_{T}^{\rho_{2}}\left(\dot{B}_{p, r}^{s_{2}}\right)},
\end{aligned}
$$


and

$$
d(\mathbb{G}(u), \mathbb{G}(v)) \lesssim \eta d(u, v) .
$$

Now by the standard contraction mapping argument ([16]), there exists a unique solution $u \in \mathscr{V}_{T}$ for $T$ small appropriately. And from Proposition 3.1, we get

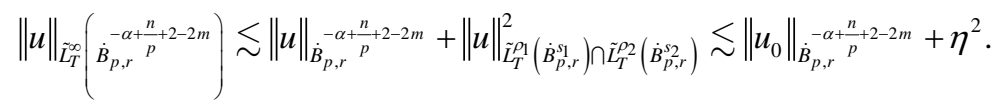

Thus the solution $u$ can be extended to the maximum time $T^{*}$, we have

$$
u \in \tilde{L}^{\infty}\left(0, T^{*} ; \dot{B}_{p, r}^{-\alpha+\frac{n}{p}+2-2 m}\right) \cap \tilde{L}^{\rho_{1}}\left(0, T^{*} ; \dot{B}_{p, r}^{s_{1}}\right) \cap \tilde{L}^{\rho_{2}}\left(0, T^{*} ; \dot{B}_{p, r}^{s_{2}}\right) .
$$

If $T^{*}<\infty$ and $\|u\|_{\tilde{L}_{T}^{\rho_{1}}\left(\dot{B}_{p, r}^{s_{1}}\right) \cap \tilde{L}_{T}^{\rho_{2}}\left(\dot{B}_{p, r}^{s_{2}}\right)}<\infty$, we need to premeditate the following integral equation

$$
u(t)=\mathrm{e}^{-(t-T) \Lambda^{\alpha}} u(T)-\int_{T}^{t} \mathrm{e}^{-(t-\tau) \Lambda^{\alpha}} \nabla \cdot\left(u \nabla(-\Delta)^{-m} u\right) \mathrm{d} \tau .
$$

Similarly, we show that

$$
\begin{aligned}
& \|u(t)\|_{\tilde{I}_{T}^{\rho_{1}}\left(T, T^{*} ; \dot{B}_{P, r}^{s_{1}}\right) \cap \tilde{L}_{T}^{\rho_{2}}\left(T, T^{*} ; \dot{B}_{P, r}^{s_{2}}\right)}
\end{aligned}
$$

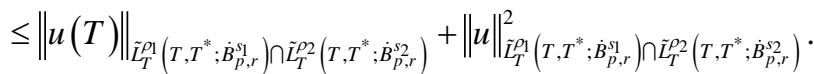

Using the contraction mapping argument as in (16), the solution exists on $\left[T, T^{*}\right]$. Taking $T$ closed to $T^{*}$ and by the local existence, the solution exists on some time larger than $T^{*}$, which contradicts the maximum time $T^{*}$. Besides, we can take $T=\infty$ in (16) and (17) if $\left\|u_{0}\right\|_{\dot{B}_{p, r}+\frac{n}{p}+2-2 m}$ is small enough. Thus we proved Theorem 3.3.

\subsection{The Case $1<\alpha<2$ and $p=\infty$ : Well-Posedness}

In this part, we study the endpoint case $p=\infty$. The crucial bilinear estimates are as follow.

Lemma 3.4 For $1<\alpha<2, m \geq 1$, we have

$$
\left\|u \nabla(-\Delta)^{-m} v\right\|_{\tilde{L}_{t}^{1}\left(\dot{B}_{\infty, 1}^{3-\alpha-2 m}\right)} \lesssim\|u\|_{\tilde{L}_{t}^{\infty}\left(\dot{B}_{\infty, 1}^{-\alpha+2-2 m)}\right)}\|v\|_{\tilde{L}_{t}^{(}\left(\dot{B}_{\infty, 1}^{2-2 m}\right)} \cdot
$$

Proof. We consider the following the estimation of $I_{1}$ :

$$
\begin{aligned}
\left\|\Delta_{j} I_{1}\right\|_{L_{t}^{1}\left(L^{\infty}\right)} & \lesssim \sum_{\left|j-j^{\prime}\right| \leq 4}\left\|\Delta_{j^{\prime}} u\right\|_{L_{t}^{1}\left(L^{\infty}\right)} \sum_{k \leq j^{\prime}-2} 2^{k(\alpha-1)} 2^{(-\alpha+2-2 m) k}\left\|\Delta_{k} v\right\|_{L_{t}^{\infty}\left(L^{\infty}\right)} \\
& \lesssim 2^{(\alpha+2 m-3) j} \sum_{\left|j-j^{\prime}\right| \leq 4} 2^{(\alpha+2 m-3)\left(j^{\prime}-j\right)} 2^{(2-2 m) j^{\prime}}\left\|\Delta_{j^{\prime}} u\right\|_{L_{t}^{1}\left(L^{\infty}\right)}\|v\|_{\tilde{L}_{t}^{\infty}\left(\dot{B}_{\infty, 1}^{-\alpha+2-2-2 m}\right)} .
\end{aligned}
$$

Therefore, according to Definition 2.2, there holds

$$
\left\|I_{1}\right\|_{\dot{L}_{t}^{1}\left(\dot{B}_{\infty, 1}^{3-2 m-\alpha}\right)} \lesssim\|u\|_{\tilde{L}_{t}^{\infty}\left(\dot{B}_{\infty, 1}^{-\alpha+2-2 m}\right)}\|v\|_{\dot{L}_{t}^{1}\left(\dot{B}_{\infty, 1}^{2-2 m}\right)} .
$$

In the same way, for $I_{2}$, when $m \geq 1$, we obtain

$$
\left\|I_{2}\right\|_{\hat{L}_{t}^{t}\left(\dot{B}_{\infty, 1}^{3-2 m-\alpha}\right)} \lesssim\|u\|_{\tilde{L}_{t}^{\infty}\left(\dot{B}_{\infty, 1}^{-\alpha+2-2 m}\right)}\|v\|_{\hat{L}_{t}^{1}\left(\dot{B}_{\infty, 1}^{2-2 m}\right)} .
$$


Now we treat with $I_{3}$.

$$
\begin{aligned}
\left\|\Delta_{j} I_{3}\right\|_{L_{t}^{1}\left(L^{\infty}\right)} & \lesssim \sum_{j^{\prime} \geq j-N_{0}} \sum_{j^{\prime}-j^{\prime \prime} \leq 1}\left\|\Delta_{j^{\prime}} u\right\|_{L_{t}^{1}\left(L^{\infty}\right)} 2^{(\alpha-1) j^{\prime \prime}} 2^{(-\alpha+2-2 m) j^{\prime \prime}}\left\|\Delta_{j^{\prime \prime}} v\right\|_{L_{t}^{\infty}\left(L^{\infty}\right)} \\
& \lesssim 2^{(\alpha+2 m-3) j} \sum_{j^{\prime} \geq j-N_{0}} 2^{(\alpha+2 m-3)\left(j^{\prime}-j\right)} 2^{(2-2 m) j^{\prime}}\left\|\Delta_{j^{\prime}} u\right\|_{L_{t}^{1}\left(L^{\infty}\right)}\|v\|_{\dot{L}_{t}^{\infty}\left(\dot{B}_{\infty, 1}^{\alpha+2-2 m}\right)} .
\end{aligned}
$$

Under the hypothesis $1 \leq \alpha<2$, we have $\alpha+2 m-3<0$. Hence considering the estimate (21), and multiplying $2^{(3-2 m-\alpha) j}$ to the resulting inequality, then taking $l^{1}$ norm yields

$$
\left\|I_{3}\right\|_{\tilde{L}_{t}^{1}\left(\dot{B}_{\infty, 1}^{3-2 m-\alpha}\right)} \lesssim\|u\|_{\tilde{L}_{t}^{\infty}\left(\dot{B}_{\infty, 1}^{-\alpha+2-2 m)}\right)}\|v\|_{\tilde{L}_{t}^{1}\left(\dot{B}_{\infty, 1}^{2-2 m}\right)} \cdot
$$

Due to (19), (20) and (22), we come to the (20). The proof of Lemma 3.9 is done.

Theorem 3.5 Let $1<\alpha<2$ and $p=\infty$. Assume that $\left\|u_{0}\right\|_{\dot{B}_{\infty, 1}^{-\alpha+2-2 m}}$ is small enough. When $3-\alpha-2 m>0$, then there is a unique solution to this system (1) that satisfies

$$
u \in \tilde{L}^{\infty}\left(0, \infty ; \dot{B}_{\infty, 1}^{-\alpha+2-2 m}\left(\mathbb{R}^{n}\right)\right) \cap \tilde{L}^{1}\left(0, \infty ; \dot{B}_{\infty, 1}^{2-2 m}\left(\mathbb{R}^{n}\right)\right) .
$$

Proof. To proving Theorem 3.5, we consider the space $\tilde{L}_{t}^{\infty}\left(\dot{B}_{\infty, 1}^{-\alpha+2-2 m}\right) \cap \tilde{L}_{t}^{1}\left(\dot{B}_{\infty, 1}^{2-2 m}\right)$. From the mapping (15), Proposition 3.1 and Lemma 3.4, one has

$$
\begin{aligned}
\|\mathbb{G}(u)\|_{\tilde{L}_{t}^{\infty}\left(\dot{B}_{\infty, 1}^{-\alpha+2-2 m}\right) \cap \tilde{L}_{t}^{1}\left(\dot{B}_{\infty, 1}^{2-2 m}\right)} & \lesssim\left\|u_{0}\right\|_{\dot{B}_{\infty, 1}^{-\alpha+2-2 m}}+\left\|u \nabla(-\Delta)^{-m} u\right\|_{\tilde{L}_{t}^{1}\left(\dot{B}_{\infty, 1}^{3-2 m-\alpha}\right)} \\
& \lesssim\left\|u_{0}\right\|_{\dot{B}_{\infty, 1}^{-\alpha+2-2 m}}+\|u\|_{\tilde{L}_{t}^{\infty}\left(\dot{B}_{\infty, 1}^{-\alpha+2-2 m}\right) \cap \tilde{L}_{t}^{1}\left(\dot{B}_{\infty, 1}^{2-2 m}\right)} .
\end{aligned}
$$

Applying the standard contraction mapping argument as before, we can show that system (1) confesses a unique solution in $u \in \tilde{L}_{t}^{\infty}\left(\dot{B}_{\infty, 1}^{-\alpha+2-2 m}\right) \cap \tilde{L}_{t}^{1}\left(\dot{B}_{\infty, 1}^{2-2 m}\right)$ if $\left\|u_{0}\right\|_{\dot{B}_{\infty, 1}^{-\alpha+2-2 m}}$ is sufficiently small.

\subsection{The Case $1<p<\infty$ : Gevrey Analyticity}

In this part, we calculate analyticity of system (1) with initial data in $\dot{B}_{p, r}^{-\alpha+2-2 m+\frac{n}{p}}$ for $1<\alpha \leq 2$ and $1<p<\infty$. At the very beginning, let's review the following three results.

Lemma 3.6 (Lemma 3.2 in [18]) We consider the operator $E_{\alpha}:=\mathrm{e}^{-\left[(t-s)^{\frac{1}{\alpha}+s^{\frac{1}{\alpha}}-t^{\frac{1}{\alpha}} \Lambda_{1}}\right.}$ for $0 \leq s \leq t$. Then $E_{\alpha}$ is either the identity operator or is the Fourier multiplier with $L^{1}$ kernel whose $L^{1}$-norm is bounded independent of $s$ and $t$.

Lemma 3.7 (Lemma 3.3 in [18]) Assume that the operator $F_{\alpha}:=\mathrm{e}^{\frac{1}{t^{\alpha}} \Lambda_{1}-\frac{1}{2} t \Lambda^{\alpha}}$ for $t \geq 0$. Then $F_{\alpha}$ is the Fourier multiplier which maps boundedly $L^{p} \rightarrow L^{p}$ for $1<p<\infty$, and its operator norm is uniformly bounded with respect to $t \geq 0$.

Lemma 3.8 (Proposition 3.5 in [15]) Let $s \in \mathbb{R}, 1<p<\infty, 1 \leq r, \rho_{1} \leq \infty$ 
and $0<T \leq \infty$. Assume that $u_{0} \in \dot{B}_{p, r}^{s}\left(\mathbb{R}^{n}\right)$ and $f \in \tilde{L}_{T}^{\rho_{1}}\left(\mathrm{e}^{t^{\frac{1}{\alpha}} \Lambda_{1}} \dot{B}_{p, r}^{s+\frac{\alpha}{\rho_{1}}}-\alpha\left(\mathbb{R}^{n}\right)\right)$. Then ( 7 ) has a unique solution $u \in \bigcap_{\rho_{1} \leq \rho \leq \infty} \tilde{L}_{T}^{\rho}\left(\mathrm{e}^{\frac{1}{t^{\alpha}} \Lambda_{1}} \dot{B}_{p, r}^{s+\frac{\alpha}{\rho}}\right)$. In addition, there exists a constant $C>0$ depending only on $\alpha$ and $n$ so that for any $\rho_{1} \leq \rho \leq \infty$, we see

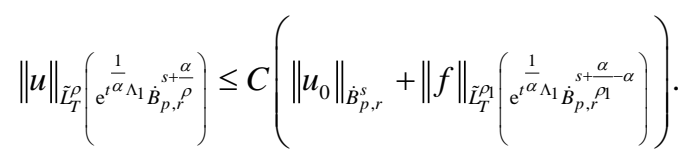

Let's take $\|f\|_{\tilde{L}_{T}^{\rho}}\left(\mathrm{e}^{\frac{1}{t^{\alpha}} \Lambda_{1} \dot{B}_{p, r}^{s}}\right)$ represent for $\left\|\mathrm{e}^{\frac{1}{t^{\alpha}} \Lambda_{1}} f\right\|_{\tilde{L}_{T}^{\rho}\left(\dot{B}_{p, r}^{s}\right)}$. Now we employ the operator $\mathrm{e}^{\frac{1}{t^{\alpha}} \Lambda_{1}}$ to certificate a result similar to Lemma 3.2.

Lemma 3.9 Let $s>0,1<p<+\infty, 1 \leq r, \rho, \rho_{1}, \rho_{2} \leq+\infty$ with $\frac{1}{\rho}=\frac{1}{\rho_{1}}+\frac{1}{\rho_{2}}$. Then for any $\varepsilon>0,0<T \leq \infty$, there holds

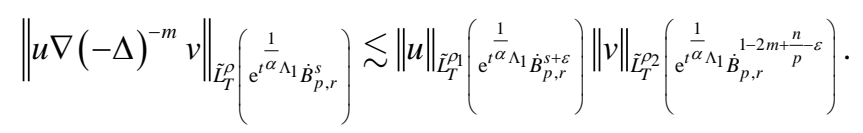

In addition, if we choose $\varepsilon=0$, then (25) also holds for $r=1$.

Proof. Set $U(t)=\mathrm{e}^{\frac{1}{t_{\alpha}} \Lambda_{1}} u(t), V(t)=\mathrm{e}^{\frac{1}{\alpha_{\alpha}} \Lambda_{1}} v(t)$. Then according to the idea of Lemma 3.2, firstly, by Bony's paraproduct decomposition to get

$$
\begin{aligned}
\mathrm{e}^{\frac{1}{\alpha^{\alpha}} \Lambda_{1}}\left(u \nabla(-\Delta)^{-m} v\right)= & \mathrm{e}^{\frac{1}{t^{\alpha}} \Lambda_{1}} \sum_{j^{\prime} \in Z} \mathrm{e}^{-t^{\frac{1}{\alpha}} \Lambda_{1}} \Delta_{j^{\prime}} U \mathrm{e}^{-t^{\frac{1}{\alpha}} \Lambda_{1}} \nabla(-\Delta)^{-m} S_{j^{\prime}-1} V \\
& +\mathrm{e}^{\frac{1}{t^{\alpha}} \Lambda_{1}} \sum_{j^{\prime} \in Z} \mathrm{e}^{-\frac{1}{\alpha} \Lambda_{1}} S_{j^{\prime}-1} U \mathrm{e}^{-\frac{1}{\alpha} \Lambda_{1}} \nabla(-\Delta)^{-m} \Delta_{j^{\prime}} V \\
& +\mathrm{e}^{t^{\frac{1}{\alpha}} \Lambda_{1}} \sum_{j^{\prime} \in Z} \sum_{j^{\prime}-j^{\prime \prime} \mid \leq 1} \mathrm{e}^{-t^{\frac{1}{\alpha}} \Lambda_{1}} \Delta_{j^{\prime}} U \mathrm{e}^{-t^{\frac{1}{\alpha}} \Lambda_{1}} \nabla(-\Delta)^{-m} \Delta_{j^{\prime \prime}} V \\
:= & J_{1}+J_{2}+J_{3} .
\end{aligned}
$$

To estimate the items $J_{i}(i=1,2,3)$, we draw support a thought in [19] and [20] and find the coming bilinear operator $\mathrm{B}_{t}(f, g)$ of the descriptor

$$
\begin{aligned}
\mathrm{B}_{t}(f, g) & :=\mathrm{e}^{t^{\frac{1}{\alpha}} \Lambda_{1}}\left(\mathrm{e}^{-t^{\frac{1}{\alpha}} \Lambda_{1}} f \mathrm{e}^{-t^{\frac{1}{\alpha}} \Lambda_{1}} g\right) \\
& =\frac{1}{(2 \pi)^{n}} \int_{\mathbb{R}^{n}} \int_{\mathbb{R}^{n}} \mathrm{e}^{i x \cdot(\xi+\eta)} \mathrm{e}^{\frac{1}{t^{\frac{1}{\alpha}}\left(|\xi+\eta|_{1}-|\xi|_{1}-|\eta|_{1}\right)}} \hat{f}(\xi) \hat{g}(\eta) \mathrm{d} \xi \mathrm{d} \eta .
\end{aligned}
$$

Based on the symbols $\xi_{j}, \eta_{j}$, and $\xi_{j}+\eta_{j}$, we can split the integration domain into subdomains. For $\zeta=\left(\zeta_{1}, \cdots, \zeta_{n}\right), \mu=\left(\mu_{1}, \cdots, \mu_{n}\right)$, $v=\left(v_{1}, \cdots, v_{n}\right) \in \mathbb{R}^{n}$ such that $\zeta_{i}, \mu_{i}, v_{i} \in\{-1,1\}$, we define

$$
D_{\zeta}:=\left\{\eta: \zeta_{i} \eta_{i} \geq 0, i=1,2, \cdots, n\right\},
$$




$$
\begin{gathered}
D_{\mu}:=\left\{\xi: \xi_{i} \mu_{i} \geq 0, i=1,2, \cdots, n\right\}, \\
D_{v}:=\left\{\xi+\eta: v_{i}\left(\xi_{i}+\eta_{i}\right) \geq 0, i=1,2, \cdots, n\right\} .
\end{gathered}
$$

$\chi_{D}$ is a characteristic function on the domain $D$. Next we redefine $\mathrm{B}_{t}(f, g)$ as

$$
\mathrm{B}_{t}(f, g):=\frac{1}{(2 \pi)^{n}} \int_{\mathbb{R}^{n}} \int_{\mathbb{R}^{n}} \mathrm{e}^{i x \cdot(\xi+\eta)} \chi_{D_{v}} \mathrm{e}^{\frac{1}{t^{\alpha}\left(\left.|\xi+\eta \eta|_{1}|| \xi\right|_{1}-|\eta|_{1}\right)}} \chi_{D_{\mu}} \hat{f}(\xi) \chi_{D_{\zeta}} \hat{g}(\eta) \mathrm{d} \xi \mathrm{d} \eta .
$$

By this way, we bring in the monodimensional operators:

$$
\begin{aligned}
& K_{1} f:=\frac{1}{2 \pi} \int_{0}^{\infty} \mathrm{e}^{i x \cdot \xi} \hat{f}(\xi) \mathrm{d} \xi, \\
& K_{-1} f:=\frac{1}{2 \pi} \int_{-\infty}^{0} \mathrm{e}^{i x \cdot \xi} \hat{f}(\xi) \mathrm{d} \xi,
\end{aligned}
$$

and

$$
\begin{aligned}
& L_{t, \varepsilon_{1}, \varepsilon_{2}} f:=f \text { if } \varepsilon_{1} \varepsilon_{2}=1, \\
& L_{t, \varepsilon_{1}, \varepsilon_{2}} f:=\frac{1}{2 \pi} \int_{-\infty}^{\infty} \mathrm{e}^{i \times \cdot \xi} \mathrm{e}^{-2 t^{\frac{1}{\alpha}}|\xi|} \hat{f}(\xi) \mathrm{d} \xi \text { if } \varepsilon_{1} \varepsilon_{2}=-1 .
\end{aligned}
$$

Furthermore for $t>0$, we have the operator

$$
Z_{t, \zeta, \mu}:=K_{\mu_{1}} L_{t, \zeta \zeta_{1}, \mu_{1}} \otimes \cdots \otimes K_{\mu_{n}} L_{t, \zeta_{n}, \mu_{n}} .
$$

The above tensor product (26) means that the $\mathrm{j}$-th operator acts on the $\mathrm{j}$-th variable of the function $f\left(x_{1}, \cdots, x_{n}\right)$. By calculation we have the following identity:

$$
\mathrm{B}_{t}(f, g)=\sum_{\zeta, \mu, v \in\{-1,1\}^{n \times 3}} K_{\mu_{1}} \otimes \cdots \otimes K_{\mu_{n}}\left(Z_{t, \zeta, \mu} f Z_{t, \zeta, \nu} g\right) .
$$

Observing that for $\xi+\eta \in D_{v}, \quad \xi \in D_{\mu}$ and $\eta \in D_{\zeta}, \mathrm{e}^{\frac{1}{t^{\alpha}}\left(|\xi+\eta|_{1}-|\xi|_{1}-|\eta|_{1}\right)}$ have to be a member of the following set:

$$
\mathbb{F}:=\left\{1, \mathrm{e}^{-2 t^{\frac{1}{\alpha}}\left|\xi_{i}+\eta_{i}\right|_{1}}, \mathrm{e}^{-2 t^{\frac{1}{\alpha}}\left|\xi_{i}\right|_{1}}, \mathrm{e}^{-2 t^{\frac{1}{\alpha}} \mid \eta_{1}}, i=1,2, \cdots, n\right\} .
$$

Notice that $\chi_{D_{\zeta}}, \chi_{D_{\mu}}, \chi_{D_{v}}$ and the elements in $\mathbb{F}$ are Fourier multipliers in $L^{p}$ for $1<p<\infty$, and the operators $K_{\mu}$ and $Z_{t, \zeta, \mu}$ are combination of identity operators and those Fourier multipliers. It is clear that the operators $K_{\mu}$ and $Z_{t, \zeta, \mu}$ are bounded linear operators on $L^{p}$ with $1<p<\infty$, and the relevant operator norm of $Z_{t, \zeta, \mu}$ is bounded independent of $t \geq 0$. Moreover, for $1<p, p_{1}, p_{2}<\infty$, we have

$$
\begin{aligned}
& \left\|\mathrm{B}_{t}(f, g)\right\|_{L^{p}} \lesssim\left\|Z_{t, \zeta, \mu} f Z_{t, \zeta, v} g\right\|_{L^{p}} \lesssim\|f\|_{L^{p_{1}}}\|g\|_{L^{p_{2}}} \\
& \text { with } \frac{1}{p}=\frac{1}{p_{1}}+\frac{1}{p_{2}} .
\end{aligned}
$$

Because of the new restriction of the bilinear operator $\mathrm{B}_{t}(f, g)$, we can do prove Lemma 3.9 based on Lemma 3.2. In fact, let's take the example of $J_{1}$ : 


$$
\begin{aligned}
\left\|\Delta_{j} J_{1}\right\|_{L_{T}^{\rho_{T}}\left(L^{p}\right)} & =\left\|\Delta_{j} \sum_{j^{\prime} \in \mathbb{Z}} \mathrm{B}_{t}\left(\Delta_{j^{\prime}} U, \nabla(-\Delta)^{-m} S_{j^{\prime}-1} V\right)\right\|_{L_{T}^{\rho}\left(L^{p}\right)} \\
& \lesssim \sum_{\left|j-j^{\prime}\right| \leq 4}\left\|K_{\mu_{1}} \otimes \cdots \otimes K_{\mu_{n}}\left(Z_{t, \zeta, \mu} \Delta_{j^{\prime}} U Z_{t, \zeta, \nu} \nabla(-\Delta)^{-m} S_{j^{\prime}-1} V\right)\right\|_{L_{T}^{\rho}\left(L^{p}\right)} \\
& \lesssim \sum_{\left|j-j^{\prime}\right| \leq 4}\left\|Z_{t, \zeta, \mu} \Delta_{j^{\prime}} U\right\|_{L_{T}^{\rho_{1}}\left(L^{p}\right)}\left\|Z_{t, \zeta, v} \nabla(-\Delta)^{-m} S_{j^{\prime}-1} V\right\|_{L_{T}^{\rho_{2}}\left(L^{\infty}\right)} \\
& \lesssim \sum_{\left|j-j^{\prime}\right| \leq 4}\left\|\Delta_{j^{\prime}} U\right\|_{L_{T}^{\rho_{1}}\left(L^{p}\right)} \sum_{k \leq j^{\prime}-2} 2^{\varepsilon k} 2^{k\left(1-2 m+\frac{n}{p}-\varepsilon\right)}\left\|\Delta_{k} V\right\|_{L_{T}^{\rho_{2}}\left(L^{p}\right)} \\
& \lesssim \sum_{\left|j-j^{\prime}\right| \leq 4} 2^{-s j^{\prime}} 2^{j^{\prime}(s+\varepsilon)}\left\|\Delta_{j^{\prime}} U\right\|_{L_{T}^{\rho_{1}}\left(L^{p}\right)}\|V\|_{\tilde{L}_{T}^{\rho_{2}}\left(\dot{B}_{p, q}^{1-2 m+\frac{n}{p}-\varepsilon}\right) .}
\end{aligned}
$$

The rest of terms are similarly estimated. Therefore, we get the estimated formula (25).

Theorem 3.10 Let $1<p<\infty$. Then the solution obtained in Theorem 3.3 satisfies

$$
\mathrm{e}^{\frac{1}{t^{\alpha}} \Lambda_{1}} u \in \mathcal{X}_{T^{*}}
$$

What is more, if $u_{0} \in \dot{B}_{p, r}^{-\alpha+\frac{n}{p}+2-2 m}\left(\mathbb{R}^{n}\right)$ is small enough, then $T^{*}=\infty$.

Proof. Taken together with Lemma 3.8 and Lemma 3.9, using the mapping (15), we get

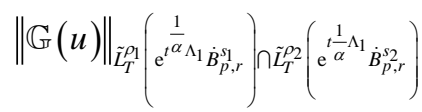

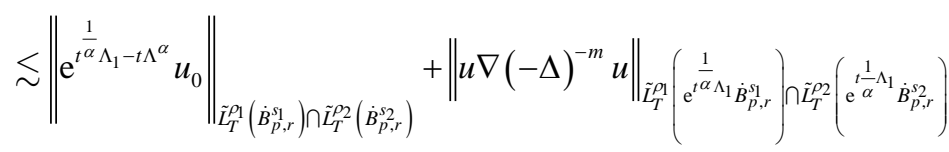

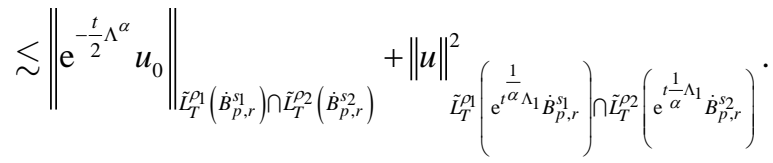

Based on estimate the above estimate and the contraction mapping argument, we accomplish the proof.

\subsection{The Case $1<\alpha<2$ and $p=\infty$ : Gevrey Analyticity}

Theorem 3.11 Let $1<\alpha<2, p=\infty$. Then the solution obtained in Theorem 3.5 satisfies

$$
\mathrm{e}^{t^{\frac{1}{\alpha}} \Lambda_{1}} u \in \tilde{L}^{\infty}\left(0, \infty ; \dot{B}_{\infty, 1}^{-\alpha+2-2 m}\left(\mathbb{R}^{n}\right)\right) \cap \tilde{L}^{1}\left(0, \infty ; \dot{B}_{\infty, 1}^{2-2 m}\left(\mathbb{R}^{n}\right)\right) .
$$

Proof. Suppose $U(t):=\mathrm{e}^{\frac{1}{t^{\alpha}} \Lambda_{1}} u(t)$. Then $U(t)$ meets the following integral equation

$$
U(t)=\mathrm{e}^{t^{\frac{1}{\alpha}} \Lambda_{1}-t \Lambda^{\alpha}} u_{0}-\int_{0}^{t}\left[\mathrm{e}^{\frac{1}{t^{\alpha}} \Lambda_{1}-(t-\tau) \Lambda^{\alpha}} \nabla \cdot\left(\mathrm{e}^{-\tau^{\frac{1}{\alpha}} \Lambda_{1}} U \cdot \mathrm{e}^{-\tau^{\frac{1}{\alpha}} \Lambda_{1}} \nabla(-\Delta)^{-m} U\right)\right] \mathrm{d} \tau .
$$


Since the symbol $e^{\frac{1}{t^{\frac{1}{\alpha}} \mid \xi}-\frac{t}{2}|\xi|^{\alpha}}$ is uniformly bounded for all $\xi$ and decays exponentially for $|\xi| \gg 1$, the Fourier multiplier $\mathbf{F}_{\alpha}:=\mathrm{e}^{t^{\frac{1}{\alpha}} \Lambda_{1}-\frac{1}{2} t \Lambda^{\alpha}}$ maps uniformly bounded from $L^{\infty}$ to $L^{\infty}$ for all $t \geq 0$. Using Young's inequality, there holds

$$
\left\|\mathrm{e}^{\frac{1}{t^{\alpha}} \Lambda_{1}-t \Lambda^{\alpha}} u_{0}\right\|_{\tilde{L}_{t}^{\infty}\left(\dot{B}_{\infty, 1}^{-\alpha+2-2 m}\right) \cap \tilde{L}_{t}^{1}\left(\dot{B}_{\infty, 1}^{-2-2 m}\right)} \lesssim\left\|\mathrm{e}^{-\frac{1}{2} t \Lambda^{\alpha}} u_{0}\right\|_{\tilde{L}_{t}^{\infty}\left(\dot{B}_{\infty, 1}^{-\alpha+2-2 m}\right) \cap \tilde{L}_{t}^{1}\left(\dot{B}_{\infty, 1}^{2-2 m}\right)} \lesssim\left\|u_{0}\right\|_{\dot{B}_{\infty, 1}^{-\alpha+2-2 m}} .
$$

For the nonlinear part, although the operators $K_{\zeta}$ and $Z_{t, \zeta, \mu}$ don't map $L^{\infty}$ to $L^{\infty}$ bounded, these operators are bounded in $L^{\infty}$ when localized in dyadic blocks. Based on the calculations line from (19) to (22) and by the continuing the same line as the proof of Lemma 3.9, and notice that Lemma 3.9, we have

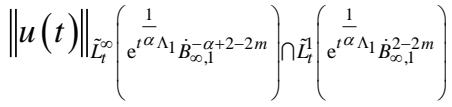

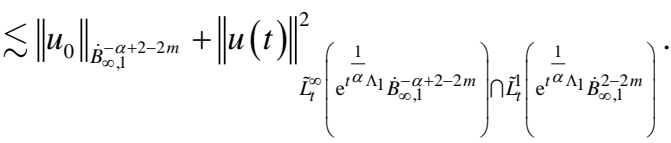

This finishes the proof, as expected.

\subsection{Decay Rate of Solution}

In this part, we concentrate on the decay rate estimates of solutions gained in Theorem 3.3, Theorem 3.5, Theorem 3.10 and Theorem 3.11. The proof is based on the following consequence.

Lemma 3.12 ([15]) For all $\sigma \geq 0$ and $1<\alpha \leq 2$, the operator $\Lambda^{\sigma} \mathrm{e}^{-\frac{1}{t^{\alpha}} \Lambda_{1}}$ is the convolution operator with a kernel $K_{\sigma}(t) \in L^{1}\left(\mathbb{R}^{n}\right)$ for all $t>0$. Moreover

$$
\left\|K_{\sigma}(t)\right\|_{L^{1}} \leq C_{\sigma} t^{-\frac{\sigma}{\alpha}},
$$

where $C_{\sigma}:=\left\|\Lambda^{\sigma} \mathrm{e}^{-\Lambda_{1}}\right\|_{L^{1}}$.

Now we have show that if the initial data $u_{0}$ is small enough in critical Besov spaces $\dot{B}_{p, r}^{-\alpha+\frac{n}{p}+2-2 m}$ for either $1<\alpha \leq 2,1<p<\infty$ and $1 \leq r \leq \infty$ or $1<\alpha<2, \quad p=\infty$ and $r=1$, then the solution is in the Gevrey class. As a result, we obtain the time decay of global mild solution for all $\sigma \geq 0$ in Besov spaces:

$$
\begin{aligned}
\left\|\Lambda^{\sigma} u(t)\right\|_{\dot{B}_{p, r}^{-\alpha+\frac{n}{p}+2-2 m}} & =\left\|\Lambda^{\sigma} \mathrm{e}^{-t^{\frac{1}{\alpha}} \Lambda_{1}} \mathrm{e}^{\frac{1}{t^{\alpha}} \Lambda_{1}} u(t)\right\|_{\dot{\dot{B}_{p, r}}-\frac{n+\frac{n}{p}+2-2 m}{}} \\
& \leq C_{\sigma} t^{-\frac{\sigma}{\alpha}}\left\|\mathrm{e}^{\frac{1}{t^{\alpha}} \Lambda_{1}} u(t)\right\|_{\dot{B}_{p, r}^{-\alpha+\frac{n}{p}+2-2 m}} \\
& \leq C_{\sigma} t^{-\frac{\sigma}{\alpha}}\left\|u_{0}\right\|_{\dot{B}_{p, r}^{-\alpha+\frac{n}{p}+2-2 m}}
\end{aligned}
$$


This proof is done.

\section{The Case $\alpha=1$ : The Proof of Theorem}

In this part, we will prove the case $\alpha=1$ for the system (1) with initial data in critical spaces $\dot{B}_{p, 1}^{1-2 m+\frac{n}{p}}(1 \leq p \leq \infty)$.

\subsection{The Case $1 \leq p<\infty$ : Well-Posedness}

For any initial data $u_{0} \in \dot{B}_{p, 1}^{1-2 m+\frac{n}{p}}$, we think the resolution space $\tilde{L}_{t}^{\infty}\left(\dot{B}_{p, 1}^{1-2 m+\frac{n}{p}}\right)$. Slightly modifying the proof Lemma 3.4, we obtain the consequence as follows.

Lemma 4.1 For any $u, v \in \tilde{L}_{t}^{\infty}\left(\dot{B}_{p, 1}^{-2 m+1+\frac{n}{p}}\right)$, when $\frac{1}{2}<m<\min \left\{\frac{1}{2}+\frac{n}{2}, \frac{1}{2}+\frac{n}{p}\right\}$, we find

$$
\left\|u \nabla(-\Delta)^{-m} v\right\|\left\|_{\tilde{L}_{t}^{\infty}}\left(-\frac{-2 m+1+\frac{n}{p}}{B_{p, 1}}\right) \lesssim\right\| u\left\|_{\tilde{L}_{t}^{\infty}}\left(\dot{B}_{p, 1}^{-2 m+1+\frac{n}{p}}\right)\right\| v \|_{\tilde{L}_{t}^{\infty}}\left(\dot{B}_{p, 1}^{-2 m+1+\frac{n}{p}}\right) .
$$

Proof. Firstly, we calculate the estimation of $I_{1}$ :

$$
\begin{aligned}
\left\|\Delta_{j} I_{1}\right\|_{L_{t}^{\infty}\left(L^{p}\right)} & \lesssim \sum_{\mid j-j^{\prime} \leq 4}\left\|\Delta_{j^{\prime}} u\right\|_{L_{t}^{\infty}\left(L^{p}\right)} \sum_{k \leq j^{\prime}-2} 2^{k\left(-2 m+1+\frac{n}{p}\right)}\left\|\Delta_{k} v\right\|_{L_{t}^{\infty}\left(L^{p}\right)} \\
& \lesssim \sum_{\left|j-j^{\prime}\right| \leq 4}\left\|\Delta_{j^{\prime}} u\right\|_{L_{t}^{\infty}\left(L^{p}\right)}\|v\|_{L_{L_{t}^{\infty}}\left(\dot{B}_{p, 1}^{-2 m+1+\frac{n}{p}}\right) .}
\end{aligned}
$$

Multiplying $2^{\left(-2 m+1+\frac{n}{p}\right) j}$ to (32), then taking $\ell^{1}$ norm to the resulting inequality, we obtain

$$
\left\|I_{1}\right\|_{\tilde{L}_{t}^{\infty}}\left(\dot{B}_{p, 1}^{-2 m+1+\frac{n}{p}}\right) \lesssim\|U\|_{\tilde{L}_{t}^{\infty}}\left(\dot{B}_{p, 1}^{-2 m+1+\frac{n}{p}}\right)\|V\|_{\tilde{L}_{t}^{\infty}}\left(\dot{B}_{p, 1}^{-2 m+1+\frac{n}{p}}\right) .
$$

Meanwhile, for $I_{2}$, we obtain

$$
\left\|I_{2}\right\|_{\tilde{L}_{t}^{\infty}}\left(\dot{B}_{p, 1}^{-2 m+1+\frac{n}{p}}\right) \lesssim\|U\|\left\|_{\tilde{L}_{t}^{\infty}}\left(\dot{B}_{p, 1}^{-2 m+1+\frac{n}{p}}\right)\right\| V \|_{\tilde{L}_{t}^{\infty}}\left(\dot{B}_{p, 1}^{-2 m+1+\frac{n}{p}}\right) .
$$

Now we treat with $I_{3}$. We discuss in two situations. One is the case of $2 \leq p<\infty$ :

$$
\begin{aligned}
& \left\|\Delta_{j} I_{3}\right\|_{L_{t}^{\infty}\left(L^{p}\right)} \lesssim 2^{\frac{n}{p} j} \sum_{j^{\prime} \geq j-N_{0}} \sum_{\mid j^{\prime}-j^{\prime \prime} \leq 1}\left\|\Delta_{j^{\prime}} u\right\|_{L_{t}^{\infty}\left(L^{p}\right)} 2^{\left(\frac{n}{p}-2 m+1\right)}\left\|j_{j^{\prime \prime}}\right\| \Delta_{j^{\prime}} v \|_{L_{t}^{\infty}\left(L^{p}\right)} \\
& \lesssim 2^{\left(2 m-1-\frac{n}{p}\right) j} \sum_{j^{\prime} \geq j-N_{0}} 2^{-\left(-2 m+1+\frac{2 n}{p}\right)\left(j^{\prime}-j\right)} 2^{\left(-2 m+1+\frac{n}{p}\right) j^{\prime}}\left\|\Delta_{j^{\prime}} u\right\|_{L_{t}^{\infty}\left(L^{p}\right)}\|v\|_{\tilde{L}_{t}^{\infty}\left(\dot{B}_{p, 1}^{-2 m+1+\frac{n}{p}}\right),}
\end{aligned}
$$

the other is that $1 \leq p<2$, and satisfies that $2 \leq p^{\prime} \leq \infty$ and $\frac{1}{p}+\frac{1}{p^{\prime}}=1$, that is 


$$
\begin{aligned}
& \left\|\Delta_{j} I_{3}\right\|_{L_{t}^{\infty}\left(L^{p}\right)} \\
& \lesssim 2^{-\left(1-2 m+\frac{n}{p}\right) j} \sum_{j^{\prime} \geq j-N_{0}} 2^{-(n-2 m+1)\left(j^{\prime}-j\right)} 2^{\left(-2 m+1+\frac{n}{p}\right) j^{\prime}}\left\|\Delta_{j^{\prime}} u\right\|_{L_{t}^{\infty}\left(L^{p}\right)}\|v\|_{\tilde{L}_{t}^{\infty}}\left(\dot{B}_{p, 1}^{-2 m+1+\frac{n}{p}}\right) .
\end{aligned}
$$

Multiplying $\left\|\Delta_{j} I_{3}\right\|_{L_{t}^{\infty}\left(L^{p}\right)}$ by $2^{\left(1-2 m+\frac{n}{p}\right) j}$ and taking $\ell^{1}$ norm to them, we get

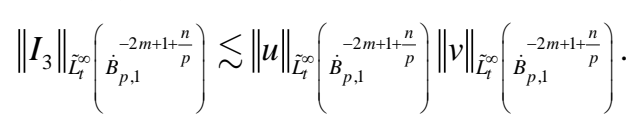

Theorem 4.2 Let $1 \leq p<\infty$. Then there exists a sufficiently small $\left\|u_{0}\right\|_{\dot{B}_{p, 1}^{p}}^{\frac{n}{p}+1-2 m}$ such that the system (1) has a unique solution $u \in \tilde{L}^{\infty}\left(0, \infty ; \dot{B}_{p, 1}^{\frac{n}{p}+1-2 m}\left(\mathbb{R}^{n}\right)\right)$.

Proof. According to the Proposition 3.1, Lemma 4.1 and the mapping (15), there holds

$$
\begin{aligned}
& \|\mathbb{G}(u)\|_{\tilde{L}_{t}^{\infty}}\left(\dot{B}_{p, 1}^{1-2 m+\frac{n}{p}}\right) \lesssim\left\|u_{0}\right\|_{\dot{B}_{p, 1}^{1-2 m+\frac{n}{p}}}+\left\|u \nabla(-\Delta)^{-m} u\right\|_{\tilde{L}_{t}^{\infty}}\left(\dot{B}_{p, 1}^{1-2 m+\frac{n}{p}}\right)
\end{aligned}
$$

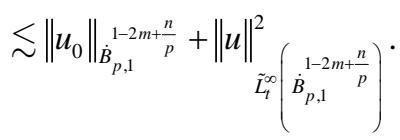

By the contraction mapping argument as before, the system (1) admits a unique solution in $\tilde{L}_{t}^{\infty}\left(\dot{B}_{p, 1}^{1-2 m+\frac{n}{p}}\right)$ for small $\left\|u_{0}\right\|_{\dot{B}_{p, 1}^{1-2 m+\frac{n}{p}}}$.

\subsection{The Case $p=\infty$ : Well-Posedness}

In the case $p=\infty$, the resolution space $\tilde{L}_{t}^{\infty}\left(\dot{B}_{\infty, 1}^{1-2 m}\right)$ is not able to be adjusted to the system (1). We use the resolution space $\tilde{L}_{t}^{\infty}\left(\dot{B}_{\infty, 1}^{1-2 m}\right) \cap \tilde{L}_{t}^{1}\left(\dot{B}_{\infty, 1}^{2-2 m}\right)$.

Theorem 4.3 Let $p=\infty$. Assume that $\left\|u_{0}\right\|_{\dot{B}_{\infty, 1}^{1-2 m}}$ is small enough. Then there is a unique solution to the system (1) that satisfies

$$
u \in \tilde{L}^{\infty}\left(0, \infty ; \dot{B}_{\infty, 1}^{1-2 m}\left(\mathbb{R}^{n}\right)\right) \cap \tilde{L}^{1}\left(0, \infty ; \dot{B}_{\infty, 1}^{2-2 m}\left(\mathbb{R}^{n}\right)\right) .
$$

Proof. By Proposition 3.1,

$$
\left\|\mathrm{e}^{-t \Lambda} u_{0}\right\|_{\tilde{L}_{t}^{\infty}\left(\dot{B}_{\infty, 1}^{1-2 m}\right) \cap \tilde{L}_{t}^{1}\left(\dot{B}_{\infty, 1}^{2-2 m}\right)} \lesssim\left\|u_{0}\right\|_{\dot{B}_{\infty, 1}^{1-2 m}} .
$$

Next, through Lemma 3.4, we obtain

$$
\left\|u \nabla(-\Delta)^{-m} u\right\|_{\tilde{L}_{t}^{1}\left(\dot{B}_{\infty, 1}^{2-2 m}\right)} \lesssim\|u\|_{\tilde{L}_{t}^{\infty}\left(\dot{B}_{\infty, 1}^{1-2 m}\right) \cap \tilde{L}_{t}^{1}\left(\dot{B}_{\infty, 1}^{2-2 m}\right)}^{2} .
$$

Therefore think over the mapping (15), we infer from Proposition 3.1, (33) and (34) that

$$
\begin{aligned}
\|\mathbb{G}(u)\|_{\tilde{L}_{t}^{\infty}\left(\dot{B}_{\infty, 1}^{1-2 m}\right) \cap \tilde{L}_{t}^{1}\left(\dot{B}_{\infty, 1}^{2-2 m}\right)} & \lesssim\left\|u_{0}\right\|_{\dot{B}_{\infty, 1}^{1-2 m}}+\left\|u \nabla(-\Delta)^{-m} u\right\|_{\dot{L}_{t}^{1}\left(\dot{B}_{\infty, 1}^{1-2 m}\right)} \\
& \lesssim\left\|u_{0}\right\|_{\dot{B}_{\infty, 1}^{1-2 m}}+\|u\|_{\tilde{L}_{t}^{\infty}\left(\dot{B}_{\infty, 1}^{1-2 m}\right) \cap \tilde{L}_{t}^{1}\left(\dot{B}_{\infty, 1}^{2-2 m}\right)}^{2}
\end{aligned}
$$


Thus by the standard contraction mapping argument, system 1 admits a unique solution in $\tilde{L}_{t}^{\infty}\left(\dot{B}_{\infty, 1}^{1-2 m}\left(\mathbb{R}^{n}\right)\right) \cap \tilde{L}_{t}^{1}\left(\dot{B}_{\infty, 1}^{2-2 m}\left(\mathbb{R}^{n}\right)\right)$ for small initial data $\left\|u_{0}\right\|_{\dot{B}_{p, 1}^{1-2 m}}$.

\subsection{The Case $1<p<\infty$ : Gevrey Analyticity}

Theorem 4.4 Let $1<p<\infty$. Then the solution acquired in Theorem 4.2 meets

$$
\mathrm{e}^{\frac{1}{t^{2 n}} \Lambda_{1}} u \in \tilde{L}^{\infty}\left(0, \infty ; \dot{B}_{p, 1}^{\frac{n}{p}+1-2 m}\left(\mathbb{R}^{n}\right)\right)
$$

Proof. The dissipation term $\mathrm{e}^{-t \Lambda}$ is not sufficiently strong to overcome the operator $\mathrm{e}^{-t \Lambda_{1}}$. Thus, we demand to define a more precise Gevrey operator. Because of $\frac{1}{2 n}|\xi|_{1}<\frac{1}{2}|\xi|$, we choose

$$
U(t):=\mathrm{e}^{\frac{1}{2 n} t \Lambda_{1}} u(t)
$$

So $U(t)$ fulfills the integral equation as follows:

$$
U(t)=\mathrm{e}^{\frac{1}{2 n} t \Lambda_{1}-t \Lambda} u_{0}-\int_{0}^{t}\left[\mathrm{e}^{\frac{1}{2 n} t \Lambda_{1}-(t-\tau) \Lambda} \nabla \cdot\left(\mathrm{e}^{-\frac{1}{2 n} \tau \Lambda_{1}} U \cdot \mathrm{e}^{-\frac{1}{2 n} \tau \Lambda_{1}} \nabla(-\Delta)^{-m} U\right)\right] \mathrm{d} \tau \text {. }
$$

Because the symbol $\mathrm{e}^{\frac{1}{2 n} t|\xi|_{1}-\frac{1}{2} t|\xi|}$ is uniformly bounded and decays for any $|\xi| \geq 1$, the operator $\mathrm{e}^{\frac{1}{2 n} t \Lambda_{1}-\frac{1}{2} t \Lambda}$ is a Fourier multiplier map $L^{p}$ to $L^{p}$ $(1<p<\infty)$ with uniformly bound independent of $t$. By Proposition 3.1, there holds

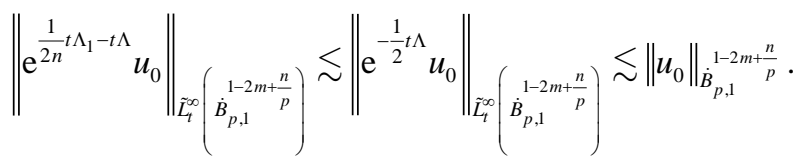

With regard to the nonlinear term, we define

$$
\mathrm{e}^{\frac{1}{2 n} t \Lambda_{1}-(t-\tau) \Lambda}=\mathrm{e}^{\frac{1}{2 n}(t-\tau) \Lambda_{1}-(t-\tau) \Lambda} \mathrm{e}^{\frac{1}{2 n} \tau \Lambda_{1}} .
$$

Hence by the new boundedness properties of the operator $e^{\frac{1}{2 n} t \Lambda_{1}-\frac{1}{2} t \Lambda}$ and the bilinear operator $\tilde{\mathcal{B}}_{t}(f, g)$ of the form

$$
\tilde{\mathcal{B}}_{t}(f, g):=\mathrm{e}^{\frac{1}{2 n} t \Lambda_{1}}\left(\mathrm{e}^{-\frac{1}{2 n} t \Lambda_{1}} f \mathrm{e}^{-\frac{1}{2 n} t \Lambda_{1}} g\right),
$$

we can get the Gevrey analyticity of the global solution as before. In fact, the corresponding operators $\tilde{K}_{\zeta}$ and $\tilde{Z}_{t, \zeta, \mu}$ are bounded independent of $t \geq 0$, hence, in regard to $1<p, p_{1}, p_{2}<\infty, \frac{1}{p}=\frac{1}{p_{1}}+\frac{1}{p_{2}}$, we have

$$
\left\|\tilde{\mathcal{B}}_{t}(f, g)\right\|_{L^{p}} \lesssim\left\|\tilde{Z}_{t, \zeta, \mu} f \tilde{Z}_{t, \zeta, v} g\right\|_{L^{p}} \lesssim\|f\|_{L^{p_{1}}}\|g\|_{L^{p^{2}}} .
$$




\subsection{The Case $p=\infty$ : Gevrey Analyticity}

In order to resolve the Gevrey analyticity of the solution in the case $p=\infty$, the following a priori estimate is true:

$$
\left\|\mathrm{e}^{\frac{1}{2 n} t \Lambda_{1}} u(t)\right\|_{\tilde{L}_{t}^{\infty}\left(\dot{B}_{\infty, 1}^{1-2 m}\right) \cap \tilde{L}_{t}^{(}\left(\dot{B}_{\infty, 1}^{2-2 m}\right)} \lesssim\left\|u_{0}\right\|_{\dot{B}_{\infty, 1}^{1-2 m}}+\left\|\mathrm{e}^{\frac{1}{2 n} t \Lambda_{1}} u(t)\right\|_{\tilde{L}_{t}^{\infty}\left(\dot{B}_{\infty, 1}^{1-2 m}\right) \cap \tilde{L}_{t}^{1}\left(\dot{B}_{\infty, 1}^{2-2 m}\right)}^{2} .
$$

Theorem 4.5 Let $p=\infty$. Then the solution is given in theorem 4.3 which satisfies

$$
\mathrm{e}^{\frac{1}{t^{2 n}} \Lambda_{1}} u \in \tilde{L}^{\infty}\left(0, \infty ; \dot{B}_{\infty, 1}^{1-2 m}\left(\mathbb{R}^{n}\right)\right) \cap \tilde{L}^{1}\left(0, \infty ; \dot{B}_{\infty, 1}^{2-2 m}\left(\mathbb{R}^{n}\right)\right) .
$$

Proof. Because from previous analysis, for $t \geq 0$, we know

$$
\left\|\mathrm{e}^{\frac{1}{2 n} t \Lambda_{1}-t \Lambda} u_{0}\right\|_{\tilde{L}_{t}^{\infty}\left(\dot{B}_{\infty, 1}^{1-2 m}\right) \cap \tilde{L}_{t}^{\underline{1}}\left(\dot{B}_{\infty, 1}^{2-2 m}\right)} \lesssim\left\|\mathrm{e}^{-\frac{1}{2} t \Lambda} u_{0}\right\|_{\tilde{L}_{t}^{\infty}\left(\dot{B}_{\infty, 1}^{1-2 m}\right) \cap \tilde{L}_{t}^{1}\left(\dot{B}_{\infty, 1}^{2-2 m}\right)} \lesssim\left\|u_{0}\right\|_{\dot{B}_{\infty, 1}^{-2-2 m}} .
$$

With respect to the nonlinear part, we find out the bilinear operator $\tilde{\mathcal{B}}_{t}(f, g)$ of the form

$$
\tilde{\mathcal{B}}_{t}(f, g)=\mathrm{e}^{\frac{1}{2 n} t \Lambda_{1}}\left(\mathrm{e}^{-\frac{1}{2 n} t \Lambda_{1}} f \mathrm{e}^{-\frac{1}{2 n} t \Lambda_{1}} g\right) .
$$

Although the above operator is not bounded from $L^{\infty} \times L^{\infty}$ to $L^{\infty}$, by the similar analysis as before, we can conclude the results line by line.

\subsection{Decay Rate of Solution}

In this part, we hold up the decay rate estimates of solutions gotten in Theorem obtained in the fourth part. On the strength of Lemma 3.12, we know that for all $\sigma \geq 0$, the operator $\Lambda^{\sigma} \mathrm{e}^{-\frac{1}{2 n} \Lambda_{1}}$ is the convolution operator with a kernel $K_{\sigma}(t) \in L^{1}\left(\mathbb{R}^{n}\right)$ for all $t>0$. What's more,

$$
\left\|K_{\sigma}(t)\right\|_{L^{1}} \leq \tilde{C}_{\sigma} t^{-\sigma}
$$

where $\tilde{C}_{\sigma}:=\left\|\Lambda^{\sigma} \mathrm{e}^{-\frac{1}{2 n} \Lambda_{1}}\right\|_{L^{1}}$. Theorem obtained in fourth part shows that if $u_{0} \in \dot{B}_{p, 1}^{\frac{n}{p}+1-2 m}$ is sufficiently small, then the solution is in the Gevrey class. As a result, for $\sigma \geq 0$, utilizing (41), we acquire

$$
\begin{aligned}
\left\|\Lambda^{\sigma} u(t)\right\|_{\dot{B}_{p, 1}^{\frac{n}{p}+1-2 m}} & =\left\|\Lambda^{\sigma} \mathrm{e}^{-\frac{1}{2 n} t \Lambda_{1}} \mathrm{e}^{\frac{1}{2 n} t \Lambda_{1}} u(t)\right\|_{\frac{n}{\dot{B}_{p, 1}^{p}+1-2 m}} \\
& \leq \tilde{C}_{\sigma} t^{-\sigma}\left\|\mathrm{e}^{\frac{1}{2 n} t \Lambda_{1}} u(t)\right\|_{\dot{B}_{p, 1}^{\frac{n}{p}+1-2 m}} \\
& \leq \tilde{C}_{\sigma} t^{-\sigma}\left\|u_{0}\right\|_{\dot{B}_{p, 1}^{\frac{n}{p}+1-2 m}}
\end{aligned}
$$




\section{Conclusions}

In summary, we can get the following conclusions:

Conclusion 5.1 Let $n \geq 2,1<\alpha \leq 2$. Suppose $u_{0} \in \dot{B}_{p, r}^{-\alpha+\frac{n}{p}+2-2 m}\left(\mathbb{R}^{n}\right)$. When $1 \leq p, r \leq \infty$, there exists the following results:

(1) (Well-posedness for $1 \leq p<\infty$ ) Let $1 \leq p<\infty, \frac{2 \alpha-2}{\alpha}=\frac{1}{\rho_{1}}+\frac{1}{\rho_{2}}$, $s=1-2 m+\frac{n}{p}$ and $\varepsilon=1-\alpha+\frac{\alpha}{\rho_{1}}$. Then we have a $T^{*}=T^{*}\left(u_{0}\right)>0$ such that the system (1) has a unique solution $u \in \mathcal{X}_{T^{*}}$, where

$$
\mathcal{X}_{T^{*}}:=\tilde{L}^{\infty}\left(0, T^{*} ; \dot{B}_{p, r}^{-\alpha+\frac{n}{p}+2-2 m}\left(\mathbb{R}^{n}\right)\right) \cap \tilde{L}^{\rho_{1}}\left(0, T^{*} ; \dot{B}_{p, r}^{s_{1}}\left(\mathbb{R}^{n}\right)\right) \cap \tilde{L}^{\rho_{2}}\left(0, T^{*} ; \dot{B}_{p, r}^{s_{2}}\left(\mathbb{R}^{n}\right)\right)
$$

with

$$
s_{1}=\frac{n}{p}-\alpha+2-2 m+\frac{\alpha}{\rho_{1}}, s_{2}=\frac{n}{p}-\alpha+2-2 m+\frac{\alpha}{\rho_{2}}, \frac{1}{\rho_{1}}+\frac{1}{\rho_{2}}=\frac{1}{\rho} .
$$

If $T^{*}<\infty$, we have

$$
\|u\|_{\tilde{L}_{T^{*}}^{\rho_{1}}\left(\dot{B}_{p, r}^{s_{1}}\right) \cap \tilde{L}_{T^{*}}^{\rho_{2}}\left(\dot{B}_{P, r}^{s_{2}}\right)}=\infty .
$$

Furthermore, if the initial value $u_{0}$ is small enough in $\dot{B}_{p, r}^{-\alpha+\frac{n}{p}+2-2 m}\left(\mathbb{R}^{n}\right)$, then $T^{*}=\infty$.

(2) (Well-posedness for $p=\infty$ ) Let $1<\alpha<2$ and $p=\infty$. Assume that $\left\|u_{0}\right\|_{\dot{B}_{\infty, 1}^{-\alpha+2-2 m}}$ is small enough. When $3-\alpha-2 m>0$, then there is a unique solution to this system (1) that satisfies

$$
u \in \tilde{L}^{\infty}\left(0, \infty ; \dot{B}_{\infty, 1}^{-\alpha+2-2 m}\left(\mathbb{R}^{n}\right)\right) \cap \tilde{L}^{1}\left(0, \infty ; \dot{B}_{\infty, 1}^{2-2 m}\left(\mathbb{R}^{n}\right)\right) .
$$

(3) (Analyticity for $1<p<\infty$ ) Let $1<p<\infty$. Then the solution obtained in (1) satisfies

$$
\mathrm{e}^{\frac{1}{t^{\alpha}} \Lambda_{1}} u \in \mathcal{X}_{T^{*}}
$$

What is more, if $u_{0} \in \dot{B}_{p, r}^{-\alpha+\frac{n}{p}+2-2 m}\left(\mathbb{R}^{n}\right)$ is small enough, then $T^{*}=\infty$.

(4) (Analyticity for $p=\infty$ ) Let $1<\alpha<2, p=\infty$. Then the solution obtained in (2) satisfies

$$
\mathrm{e}^{\frac{1}{t^{\alpha}} \Lambda_{1}} u \in \tilde{L}^{\infty}\left(0, \infty ; \dot{B}_{\infty, 1}^{-\alpha+2-2 m}\left(\mathbb{R}^{n}\right)\right) \cap \tilde{L}^{1}\left(0, \infty ; \dot{B}_{\infty, 1}^{2-2 m}\left(\mathbb{R}^{n}\right)\right) .
$$

(5) (Decay rate for $1<p \leq \infty$ ) With any $\sigma \geq 0,1<p<\infty$ or $p=\infty$ and $r=1$, the global solution acquired in (1) and (2) satisfies

$$
\left\|\Lambda^{\sigma} u(t)\right\|_{\dot{B}_{p, r}-\alpha+\frac{n}{p}+2-2 m} \leq C_{\sigma} t^{-\frac{\sigma}{\alpha}}\left\|u_{0}\right\|_{\dot{B}_{p, r}-\frac{}{-\alpha+\frac{n}{p}+2-2 m}},
$$

where $C_{\sigma}:=\left\|\Lambda^{\sigma} \mathrm{e}^{-\Lambda_{1}}\right\|_{L^{1}}$.

Corresponding to Conclusion 5.1, when $\alpha=1$, we can get the results as follows. 
Conclusion 5.2 Let $n \geq 2, \alpha=1$ and suppose $u_{0} \in \dot{B}_{p, 1}^{\frac{n}{p}+1-2 m}\left(\mathbb{R}^{n}\right)$. When $1 \leq p \leq \infty, \frac{1}{2}<m<\min \left\{\frac{1}{2}+\frac{n}{2}, \frac{1}{2}+\frac{n}{p}\right\}$, there exists results as follows:

(1) (Well-posedness for $1 \leq p<\infty$ ) Let $1 \leq p<\infty$. Then there exists a sufficiently small $\left\|u_{0}\right\|_{\dot{B}_{p, 1}^{\frac{n}{p}}}^{\frac{n}{p}-2 m}$ such that the system (1) has a unique solution $u \in \tilde{L}^{\infty}\left(0, \infty ; \dot{B}_{p, 1}^{\frac{n}{p}+1-2 m}\left(\mathbb{R}^{n}\right)\right)$.

(2) (Well-posedness for $p=\infty$ ) Let $p=\infty$. Assume that $\left\|u_{0}\right\|_{\dot{B}_{\infty, 1}^{1-2 m}}$ is small enough. Then there is a unique solution to the system (1) that satisfies

$$
u \in \tilde{L}^{\infty}\left(0, \infty ; \dot{B}_{\infty, 1}^{1-2 m}\left(\mathbb{R}^{n}\right)\right) \cap \tilde{L}^{1}\left(0, \infty ; \dot{B}_{\infty, 1}^{2-2 m}\left(\mathbb{R}^{n}\right)\right) .
$$

(3) (Analyticity for $1<p<\infty$ ) Let $1<p<\infty$. Then the solution acquired in (1) meets

$$
\mathrm{e}^{\frac{1}{t^{2 n}}} \Lambda_{1} u \in \tilde{L}^{\infty}\left(0, \infty ; \dot{B}_{p, 1}^{\frac{n}{p}+1-2 m}\left(\mathbb{R}^{n}\right)\right)
$$

(4) (Analyticity for $p=\infty$ ) Let $p=\infty$. Then the solution is given in (2) which satisfies

$$
\mathrm{e}^{t^{\frac{1}{2 n}} \Lambda_{1}} u \in \tilde{L}^{\infty}\left(0, \infty ; \dot{B}_{\infty, 1}^{1-2 m}\left(\mathbb{R}^{n}\right)\right) \cap \tilde{L}^{1}\left(0, \infty ; \dot{B}_{\infty, 1}^{2-2 m}\left(\mathbb{R}^{n}\right)\right) .
$$

(5) (Decay rate for $1<p \leq \infty$ ) With any $\sigma \geq 0,1<p<\infty$ or $p=\infty$ and $r=1$, the global solution got in (1) and (2) fits

$$
\left\|\Lambda^{\sigma} u(t)\right\|_{\dot{B}_{p, 1}^{\frac{n}{p}+1-2 m}} \leq \tilde{C}_{\sigma} t^{-\sigma}\left\|u_{0}\right\|_{\dot{B}_{p, 1}^{\frac{n}{p}+1-2 m}},
$$

where $\tilde{C}_{\sigma}:=\left\|\Lambda^{\sigma} \mathrm{e}^{-\frac{1}{2 n} \Lambda_{1}}\right\|_{L^{1}}$

\section{Acknowledgements}

The author would like to thank collaborator, editors and reviewers for many helpful discussions and suggestions. This work was partially supported by National Natural Science Foundation of China (No. 11601223).

\section{Conflicts of Interest}

The authors declare no conflicts of interest regarding the publication of this paper.

\section{References}

[1] Feng, Q. and Liu, A. (2019) Oscillation for a Class of Fractional Differential Equation. Journal of Applied Mathematics and Physics, 7, 1429-1439. https://doi.org/10.4236/jamp.2019.77096

[2] Keller, E.F. and Segel, L.A. (1970) Initiation of Slime Mold Aggregation Viewed as 
an Instability. Journal of Theoretical Biology, 26, 399-415. https://doi.org/10.1016/0022-5193(70)90092-5

[3] Biler, P. and Karch, G. (2010) Blowup of Solutions to Generalized Keller-Segel Model. Journal of Evolution Equations, 10, 247-262.

https://doi.org/10.1007/s00028-009-0048-0

[4] Biler, P. and Wu, G. (2009) Two-Dimension Chemotaxis Models with Fractional Diffusion. Mathematical Methods in the Applied Sciences, 32, 112-126. https://doi.org/10.1002/mma.1036

[5] Zhao, J., Liu, Q. and Cui, S. (2013) Existence of Solutions for the Debye-Hückel System with Low Regularity Initial Data. Acta Applicandae Mathematicae, 125, 1-10. https://doi.org/10.1007/s10440-012-9777-0

[6] Vázquez, J.L. (1974) The Porous Medium Equation: Mathematical Theory. Oxford University Press, New York.

[7] Caffarelli, L. and Vázquez, J.L. (2010) Nonlinear Porous Medium Flow with Fractional Potential Pressure. Archive for Rational Mechanics and Analysis, 202, 537-565. https://doi.org/10.1007/s00205-011-0420-4

[8] Caffarelli, L. and Vázquez, J.L. (2016) Regularity of Solutions of the Fractional Porous Medium Flow with Exponent $\frac{1}{2}$. St. Petersburg Mathematical Journal, 27, 437-460. https://doi.org/10.1090/spmj/1397

[9] Serfaty, S. and Vázquez, J.L. (2014) A Mean Filed Equation as Limit of Nonlinear Diffusions with Fractional Laplacian Operators. Calculus of Variations and Partial Differential Equations, 49, 1091-1120. https://doi.org/10.1007/s00526-013-0613-9

[10] Foias, C. and Temam, R. (1989) Gevrey Class Regularity for the Solutions of the Navier-Stokes Equations. Journal of Functional Analysis, 87, 359-369.

https://doi.org/10.1016/0022-1236(89)90015-3

[11] Foias, C. and Temam, R. (1979) Some Analytic and Geometric Properties of the Solutions of the Evolution Navier-Stokes Equations. Journal de Mathematiques Pures et Appliquees, 58, 339-368.

[12] Zhan, M. (2001) Gevrey Class Regularity for the Solutions of the Phase-Lock Equations of Superconductivity. 406-415.

[13] Ferrari, A.B. and Titi, E.S. (1998) Gevrey Regularity for Nonlinear Analytic Parabolic Equations. Communications in Partial Differential Equations, 23, 424-448. https://doi.org/10.1080/03605309808821336

[14] Chueshov, I., Polat, M. and Siegmund, S. (2002) Gevrey Regularity of Global Attractor for Generalized Benjamin-Bona-Mahony Equation. Institute for Mathematics \& Its Applications, 22, 226-242.

[15] Zhao, J. (2018) Well-Posedness and Gevrey Analyticity of the Generalized Keller-Segel System in Critical Besov Spaces. Annali di Matematica, 197, 521-548. https://doi.org/10.1007/s10231-017-0691-y

[16] Lemarié-Rieusset, P.-G. (2002) Recent Developments in the Navier-Stokes Problem. Research Notes in Mathematics, Chapman \& Hall/CRC, Boca Raton. https://doi.org/10.1201/9781420035674

[17] Wu, G. and Yuan, J. (2008) Well-Posedness of the Cauchy Problem for the Fractional Power Dissipative Equation in Critical Besov Spaces. Journal of Mathematical Analysis \& Applications, 340, 1326-1335. https://doi.org/10.1016/j.jmaa.2007.09.060

[18] Bae, H., Biswas, A. and Tadmor, E. (2013) Analyticity of the Subcritical and Critical Quasi-Geostrophic Equations in Besov Spaces. 
[19] Bae, H., Biswas, A. and Tadmor, E. (2012) Analyticity and Decay Estimates of the Navier-Stokes Equations in Critical Besov Spaces. Archive for Rational Mechanics and Analysis, 205, 963-991. https://doi.org/10.1007/s00205-012-0532-5

[20] Lemarié-Rieusset, P.-G. (2000) On the Analyticity of Mild Solutions for the Navier-Stokes Equation. Comptes Rendus de PAcadémie des Sciences Series I, 330, 183-186. https://doi.org/10.1016/S0764-4442(00)00103-8 